Bol. Soc. Geol. Mexicana XXXV: 29-37, 1974.

\title{
Phylloceras del Cretácico inferior de San Juan Raya-Zapotitlán, Estado de Puebla. Méx.
}

Celestina González-Arreola

Investigador de Tiempo Completo

Instituto de Geología. U.N..AM

\section{RESUMEN}

Se hace un estudio taxonómico detallado de $P$. rioi (Nyst y Galeotti, 1840) basado sobre el material proveniente de la región fosilífera del Aptiano de San Juan Raya y Zapotitlán, en el Estado de Puebla. En el presente trabajo se aclara el status taxonómico de P. rioi, la cual había permanecido fuera de la nomenclatura, debido a la carencia de una revisión taxonómica detallada. Se demuestra que $P$. onoense (Stanton) 1895 es un sinónimo secundario.

\section{ABSTRACT \\ A detailed toxonomic study of $P$. rioi (Nyst y Galeotti, 1840), is given based on the material from the Aptian fossiliferous localities near San Juan Raya and Zapotitlán, Puebla. \\ $1 \mathrm{t}$ is suggested that $P$. onoense (Stanton) 1895, is a junior synonym of $P$. rioi. However $P$. rioi has been left out of the nomenclature because the lack of detailed taxonomic studies.}

\section{INTRODUCCION}

La región fosilífera de San Juan Raya y Zapotitlan está situada aproximadamente a 39 ó 40 kilómetros al Suroeste de la Ciudad de Tehuacán, en el Sureste del Estado de Puebla (Fig. 1). Es conocida desde el siglo pasado, por haber sido la localidad fosilífera de donde se describen los primeros fósiles de México, por los geólogos belgas Nyst y Galeotti (1840) quienes le atribuyeron una edad jurásica.

De esta misma localidad, el paleontólogo francés D'Orbigny $(1850$, p. 231, 240, 257) estudió algunos fósiles y los atribuyó al Senoniano. Los estudios sobre equinoides llevados a cabo por Desor (18551858) en la misma localidad le permitieron atribuirle una edad jurásica. No obstante, es de recordar que fue Coquand (1869, p. 163), quien primeramente les dió la edad correcta a los sedimentos que afloran en San Juan Raya, determinando el material de ostreidos y asignándolos al "U-rgo-Aptiano". Otros investigadores como Aguilera, Ordóñez y Buelna (1896) publicaron un bosquejo geológico de la región de San Juan Raya y Zapotitlán, donde hicieron observaciones de fauna dispersa en el suelo y atribuyen a ese material edad cretácica inferior. Más tarde, Aguilera (1906) elaboró un trabajo geológico detallado de esta misma región donde incluyó una lista de especies y efectuó comparaciones con especies europeas con las que aquellas fueron comparadas; este mismo autor, hizo mención de un estudio paleontológico realizado por él mismo y que desgraciadamente jamás fue publicado (op. cit. nota al pie del cuadro No. 1). 


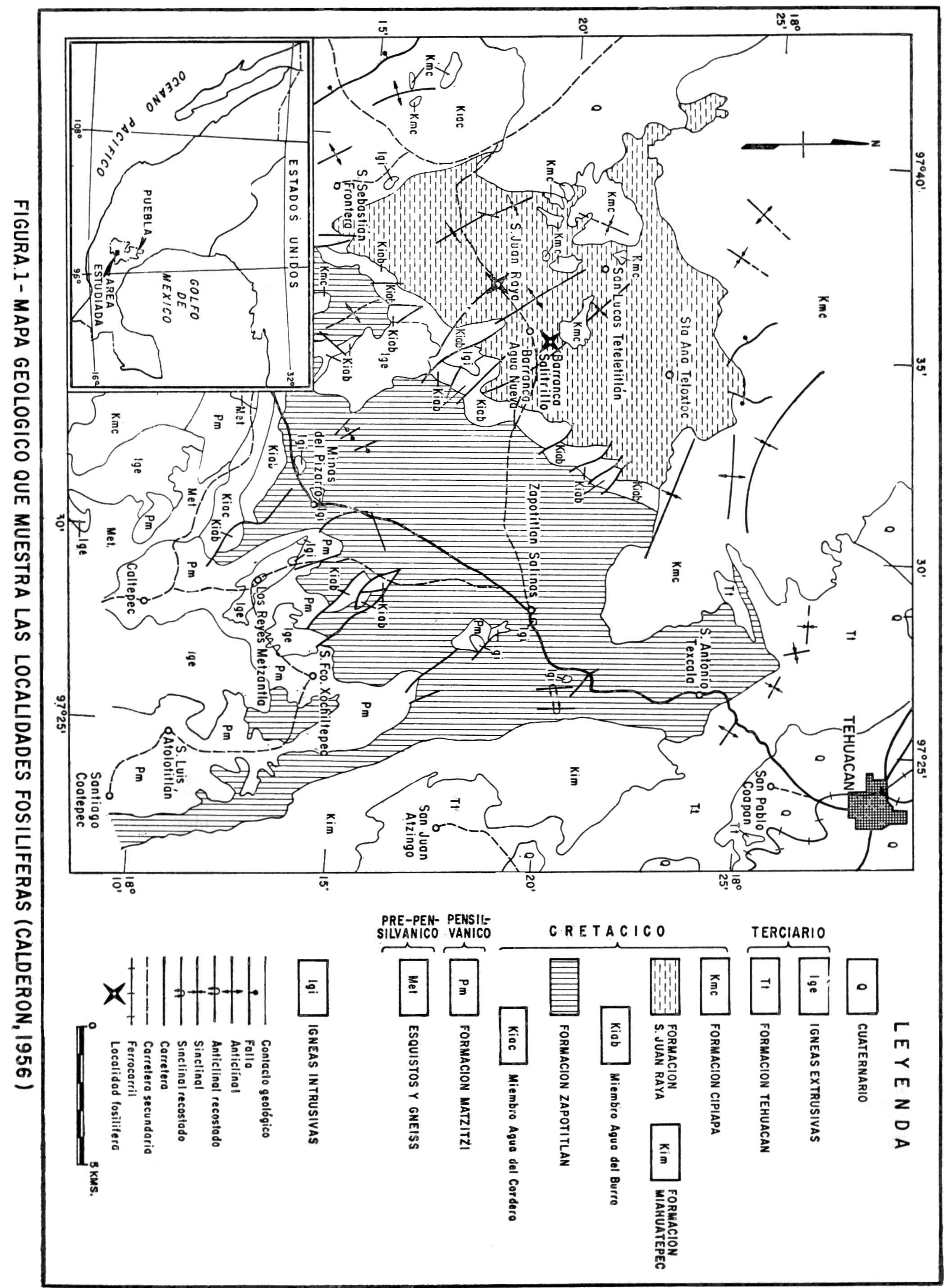


Más tarde, Burckhardt (1930, cuadro No 13) consideró que las capas de las Formaciones San Juan Raya y Zapotitlán, del Suroeste de Tehuacán Pue., pertenecen la primera al Aptiano Superior, por la presencia de Pseudocidaris galeottii Desor, Dipopodia aff. malbosi Desor, Ostrea acuticosta (Nyst y Galeotti). Trigonia plicatocostala (Nyst y Galeotti), Phyilloceras rioi (Nyst y Galeotti) y Lytoceras reconditum (Nyst y Galeotti). Respecto a la Formación Zapotitlán, el mismo autor le atribuye una edad barremiana, por haberse encontado en ella, Thamnastrea cf. stricta (Fromentel) Monopleura tulae Felix. Nerinea titania Félix y otras especies.

Posteriormente, Müllerried (1933, p. 84) estudió el material proveniente de la región antes mencionada y le asignó una edad aptiana superior, por la presencia del género Tetragonites. Además (Müllerried 1933, p. 93), identificó Phylloceras rioi (Nyst y Galeotti), aff. velladae Michelin, Lytoceras reconditum (Nyst y Ga1eotti) etc. En trabajos posteriores Müllerried (1934) continuó estudiando el Cretácico Inferior de la localidad antes mencionada, donde subdividió las rocas del Barremiano, y aquellas que las sobreyacen, de acuerdo a los rudistas, corales y amonitas.

En años más recientes se han publicado otros trabajos paleontológicos y geológicos de la región fosilífera, así Maldonado-Koerdell (1953) al estudiar los equinoides de esa región sugiere que pertenecen al Aptiano.

Calderón (1956, p. 19) estudió la Formación San Juan Raya y tomó en cuenta los trabajos paleontológicos, publicados anteriormente, con los cuales consideró que esta unidad litoestratigráfica pertenece al Aptiano. Además redescribió la Formación Zapotitlán, en el Sur de Tehuacán, Pue., que contiene una cantidad considerable de monopleuras, gasteró-. podas, amonitas muy mal conservados y braquiópodos y sugirió una edad·barremiana.

Alencáster (1956) estudió los gasterópodos y pelecípodos de la selección de Aguilera, depositada en el Museo de Paleontología del Instituto de Geología y determinó una edad aptiana y barremiana, para las Formaciones San Juan Raya y Zapotitlán. Más tarde, Reyeros (1963) estudió corales del Aptiano de la misma localidad. Por su parte Buitrón (1970) estudió los equinoides de las Formaciones San Juan Raya y Zapotitlán.

El presente trabajo constituye una revisión de los
Phylloceras de la colección del Museo de Paleontología del Instituto de Geología, colectados por Mülleried (1933); de los cuales se proporcionan todas las ilustraciones que se consideró conveniente así como sus descripciones. Aunque el estado de preservación de estos fósiles es bastante pobre, el material que se ilustra comprende estados juveniles y adultos, preservados como moldes y fragmentos de fragmacono, con parte de la última vuelta.

El género Phylloceras, es de distríbución estratígráfica bastante amplia. Otros qrupos de amonitas de la región de San Juan Raya parecen escasos; no obstante, son los fósiles ideales para situar en el tiempo, los estratos donde han sido colectados.

Sin embargo, no se está en posibilidad de señalar, la relación estratigráfica que conservan los fósiles, de la colección del Instituto de Geología, con el material descrito por Cotteau (1890), Burckhardt (1930), Müllerried (1933, 1934).

\section{AGRADECIMIENTOS}

Al Dr. Abelardo Cantú del Instituto Mexicano del Petróleo, por la valiosa ayuda en la correlación y crítica del manuscrito, así como su magnífica y desinteresada orientación.

A la Dra. Gloria Alencáster del Instituto de Geología, de la Universidad Nacional de México; al Dr. Gerd Westermann de McMaster Uníversity, Hamilton, Ontario, Canadá, y al Dr. Alberto C. Riccardi, del Museo de la Facultad de Ciencias Naturales de la Universidad Nacional de la Plata, Argentina; a todos ellos, se les agradece sinceramente por la revisión, crítica y valiosas sugerencias, que ayudaron mucho para concluir esta publicación.

Al Dr. Peter U. Badda, de California Academy of Sciences, de San Francisco, California, al Dr. J. Wyatt Durham, de University of California, Berkeley, por las facilidades que proporcionaron para tener acceso a las colecciones y facilitar material de museo, en calidad de préstamo, con el fin de compara el materíal-en estudio y se agredece también sinceramente, al Sr. Armando Altamira, por su colaboración, en haber fotografiado el material ilustrado.

ESTRATIGRAFIA DEL AREA DE TEHUACAN, PUEBLA

A pesar de desconocer la localización exacta del material paleontológico, que sirvió para realizar el 
presenle trabajo, se considera pertinente incluir la caracterización de las unidades litoestratigráficas de esa región, como se conoce actualmente (Fig. 1),

La Formación Zapotitlán recibe el nombre del Cerro Zapotitlán (Aguilera, 1906), y se caracteriza por "lutitas calcáreas grises micacíferas cor intercalaciones de margas grises" de un espesor de 100 metros (Calderón 1956, p, 16). Calderón (p. 17) ha suhdividido esta Formación en dos miembros: Agua del Cordero del Barremiano Inferior y Superior y Agua del Burro del Barremiano Superior.

La Formación Zapotitlán descansa sohre las rocas metamórficas del Complejo Basal y rocas continentales, y está cubierta concordantemente por la Formación San Juan Raya.

La Formación San Juan Raya fue reconocida originalmente por Aguilera, (1906, p .18) y la denominó "División San Juan Raya". Calderón (1956, p. 18) la denomina formación y enmienda la descripción de Aguilera.

De acuerdo con la definición dada por Calderón la Formación San Juan Raya se caracteriza por ... "lutitas de color gris verdoso, que al intemperizar cambian a verde amarillento calcárea, coquina de ostreidos"; y su espesor total se desconoce, sin embargo en una sección medida por Calderón (1956, p. 18) en el Cerro Gavilán, alcanza un espesor de 1250 metros.

Esta formación descansa concordantemente sobre la Formación Zapotitlán; su límite superior se desconoce, por estar descansando, discordantemente sobre ella las calizas de la Formación Cipiapa del Albiano Inferior (Calderón 1956, p. 22).

\section{PALEONTOLOGIA SISTEMATICA}

El material estudiado se encuentra depositado en el Museo de Paleontología del Instituto de Geología, en la Ciudad Universitaria, Los ejemplares están registrados con su número correspondiente al catálogo, con las iniciales IGM (Instituto de Geología).

Phylum: MOLLUSCA

Clase: CEPHALOPODA

Orden: A MONOIDE A ZitteL 1884

Familia: PYLLOCERATIDAE Zittel. 1884

Género: $\quad$ Suess. 1865

Hypophylloceras Salfeld. 1924
Especie Tipo: Phyiloceras onoense Staton, $1896=$ Phylloceras (Hipophylloceras) velledae (Michelin, 1834).

Phylloceras (Hipophylloceras) veledae (Michelin, 1834).

\author{
(Lámina 1, Figura 1)
}

1834 Ammonites velledae Michelin, lám. 35.

1840 Ammonites rioi Nyst y Galeotli, p. 217-218, fig. 8.

1841 Ammonites velledae D'Orbigny, p. 280, lám. 82, fig. $1-4$.

1864 Ammonites ramosus Gabh, v.1, 65, lám. 11, (nom Meek) figs. 12-12a; lám. 12, fig. $12 b$.

1895 Phyiloceras onoense Stanton, p. 74.

1898 Phyiloceras onoënsis Stanton, Smith, p. 147-149, lám. 19-20.

1906 Phylloceras rioi (Nyst y Galeotti); Aguilera, p, 15 y

1923 Phylloceras rioi (Nyst y Galeotti); Böse, p. 126.

1924 Hypophylloceras onoense Salfeld. p. 6 (fide Packard. 1960, p. 422).

1927 Hypophylloceras onoense Stanton; Spath, p. 38. cuadro.

1930 Phylloceras rioi (Nyst y Galeotti); Burckhardt, p. 159 y cuadro 13.

1933 Phylloceras rioi (Nyst y Galeotti), Müllerried, p. 86, fig. $7-10$.

1938 Phylloceras onoense Stanton; Anderson, p. 142, lám. t1, fig. $1-2$.

1943 Phylloceras onoense Stanton; Hanna y Hertlein, p. 165185, lám. 169, fig. 19.

1948 Hypophyiloceras onoense Salfeld 1924; Collignon, p. 68.

1956 Phylloceras onoense Stanton: Murphy, p. 2112-2116. fig. text. 6.

1957 Hypophylloceras onoense (Stanton): Wright in Arkell el al., p. L 189, fig. 219.

1959 Hypophyllocerw; onoense (Stanton); Matsumoto, p. 4, lám. 1, fig. 2, lám. 2, fig, 1.

1960 Hypophylloceras onoense (Stanton); Packard, p. 422, fig. text. 1·2, lám. 55, fig. 1-4, lám. 56, fig. 1.

1963 Phylloceras (Hypophylloceras) onoense Stanton; Wiedmann; p. 167, 204, 213.

Material:

En la colección del Instituto de Geología se conservan fragmentos deformados, que se incluyen en la especie que se está describiendo. Unicamente se cuenta con un eiemplar completo, hipotipo IGM-2530, fragmento donde se observa parte de la sutura. IGM-2528, sección de vuelta IGM 2534 y IGM2533, hipotipos IGM-2531, donde se observan las estrías finas y sinuosas.

Descripción:

La mayor parte de los ejempllares se encuentran comprimidos y muy mal conservados; sin embargo 
el más completo (Lám. 1, Fig. 1) tiene concha involuta, sección de vuelta ovalada, más alta que ancha. Los flancos son ligeramente convexos y la región ventral es también convexa. La ornamentación consiste de numerosas estrías, sinuosas y muy finas; en la cercanía de la región umbilical están muy juntas entre sí (Lám. 1, Fig. 5), pero a medida que se alejan de esta zona, y hacia la región lateral externa se van separando y atraviesan la región ventral sin interrupción. El número de estrías por centimetro es de 11 a 12, contadas sobre la región ventral (Lám. 1, Fig. 3).

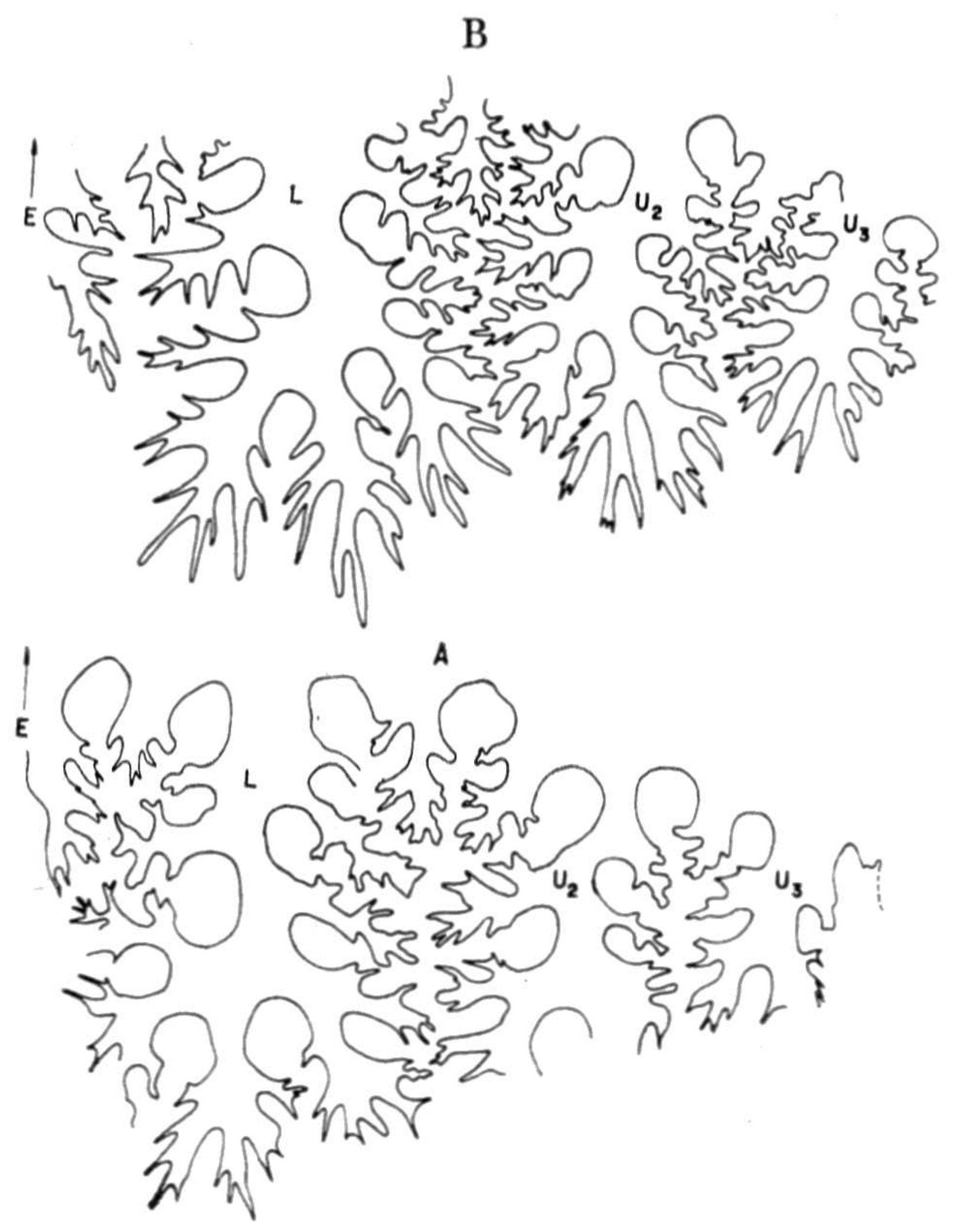

Figura 2A-B-Comparación de la línea lobular entre: A) Phylloceras (Hypophylloceras) velledae (Michelin 1834) Ejemplar IGM-2532; y B) Phylloceras onoense Stanton Hipotipo No. 7736, (C.A.S.).

La sutura no pudo ser observada en el ejemplar completo ni en los fragmentos anterionnente descritos; sin embargo, en la misma colección, se presentan otros fragmentos procedentes de la misma localidad donde este carácter es muy visible; en efecto, la sutura (Fig. 2A) es de tipo filoide, el lóbulo E. bifurcado es un poco más corto que el primer lóbulo L, Que es bastante esbelto, asimétrico y trifurcado en su base por dos pequeños elementos inclinados; U2 es ligeramente más estrecho y más corto que L y es irregularmente bifido en su base: U3 es angosto y más pequeño que U2 y está bifurcado simétricamente en su base. En otro fragmento (Lám. 1, Fig. 8) la sutura presenta el mismo número de elementos y se observa la silla E/L bifurcada asimétricamente, la silla L/U2 angosta y bifurcada y más pequeña que $\mathrm{E} / \mathrm{L}$.

Dimensiones (en $\mathrm{mm}$ )

Ejemplar IGM 2530

Diámetro de vuelta $=103.3$

Ancho de vuelta $\quad=36.4$

Altura de vuelta $\quad=59.8$

\section{OBSERVACIONES}

Los ejemplares estudiados han sido comparados con las ilustraciones de Nyst y Galeotti (1840, p. $217-$ 218, fig. 8) correspondientes a Ammonites rioi. Presentan bastante semejanza como son la involución de la concha y la sección de vuelta, la ornamentación y el número de estrías por centímetro son los mismos. Sin embargo, las estrías que se observan en la ilustración de Nyst y Galeotti (1840, fig. 8) presentan un aspecto recto, a diferencia de las de los ejemplares aquí estudiados, donde las estrías son muy finas y ligeramente sinuosas e inclinadas hacia adelante en la parte externa de la concha.

Los ejemplares arriba descritos, también fueron estudiados por Müllerried (1933, p. 86, 90) algunos ejemplares los clasificó como Phylloceras rioi y otros como Phylloceras aff. velledae Michelin. De esta última especie mencionó algunas diferencias con respecto a Phylloceras rioi "... parece tener diámetro mayor, es más redondeado del lado exterior, la satura es más complicada y tiene más sillas auxiliares que la especie rioi...", todas estas diferencias, no están representadas en la publicación antes mencionada, por lo que al examinar los ejemplares fueposible rehacer la comparación.

Cabe mencionar que Böse (1923, p. 126) hizo un comentario acerca de Phylloceras rioi y la comparó con Phylloceras velledae (Michelin, 1834) colectada del Vraconiano de Camacho, Zacatecas y consideró que no había diferencias suficientes para separar esas dos especies, por lo que sugirió que debería desaparecer el nombre especifico de rioi. Además, agregó que las capas de San Juan Raya, de donde provenía $P$. rioi pertenecen probablemente a la parte superior del Aptiano, en cambio P. velledae de Camacho, 
Zacatecas, caracteriza al Albiano Superior ( = Vraconiano) .

No obstante, se ha creido pertinente comparar los amonitas de este estudio, que a su vez fueron descritos previamente por Mülleried (1933, p. 86, 90) con algunas especies de California, por su gran semejanza. En efecto, el ejemplar de la Lam. 1, Fig. 1, se comparó con el Hipotipo No. 7736 de Philloceras onoense Stanton depositado en la colección de Paleontologia del Museo de la Academia de Ciencias de San Francisco, California, e ilustrado por Anderson (1938, p. 142, Lám. 11, Fig. 1, 2) y se encontró que ambos ejemplares son semejantes en su mor. fología; no obstante, el ejemplar de California presenta una mínima diferencia, ya que posee 13 estrías por centímetro sobre la región ventral; en cambio Phylloceras rioi (Lám. 1, Fig. 1) presenta 11-12 estrías por centímetro, en la misma área.

El fragmento ilustrado en la Lám. 1, Fig. 8, también fue comparado con el Lectotipo No. 12110 de Hypophylloceras onoense (Stanton) depositado en el Museo de la Universidad de California e ilustrado por Packard (1960, p. 422, fig. text 1, Lám. 55, figs. 1-4) y se encontró que son semejantes, únicamente se presenta una pequeña diferencia en la sutura: el lóbulo E es ligeramente cuadrado en $H$. onoense mientras que es alargado en el de México (fig. 2A-B).

Tomando en cuenta las diferencias que existen entre $H$. onoense y $P$. rioi y observando que no son significativas cabe señalar que $P$. rioi (Nyst y Galeotti 1840, p. 217-218, fig. 8) tiene prioridad sobre $H$. onoense (Stanton), por lo que se considera como sinónimo primario de esta última especie.

Se recuerda que en un trabajo publicado por Wiedmann (1963, p. 224·) relacionado con los Phylloceratinae del Cretácico Inferior de Mallorca; en donde describe especies pertenecientes a la familia Phylloceratidae, expone que las diferencias entre las suturas de Phylloceras seresitense, $P$. onoense y $P$. velledae respectivamente, son tan insignificantes que no permiten separarlos en especies diferentes: más bien opina que es necesario agrupar estos dos últimas especies en una sola. También observa que el desarrollo de la sutura de $P$. velladae es muy semejante a $P$. onoense pues en ambas especies las sillas principales se presentan difílicas en la juventud. Más tarde Wiedmann (1963, p. 213) recomienda mejor reunir los Phyloceras del Cretácico, que son muy semejantes en su sutura.

\section{DISTRIBUCION ESTRATIGRAFICA}

Phylloceras roi fue considerado del Jurásico Superior (Nyst y Galeotti 1840, p. 2); posteriormente Aguilera (1906, p. 20) a los cafalopodos de la "División San Juan Raya", los asigna al Neocomiano junto con la fauna acompañante, ya que la comparó con otra del mismo piso descrita por Pictet y Renevier (1858) procedente de la Montaña de Voirons, al este de Ginebra, Suiza.

Más tarde, Burckhardt (1930, p. 58) rectificó a Nyst y Galeotti (1840) y atribuyó la fauna colectada en San Juan Raya, al Cretácico Inferior; Müllerried $(1933$, p. 86, 93) por su parte, asignó a Phylloceras rioi al Aptiano Superior.

Los ejemplares en que se basa el trabajo de Mülleried arriba mencionado fueron comparados en este texto con Hypophylloceras onoense (Stanton), que procede de rocas del Cretácico Inferior del Norte de Califomia y cuyo rango estratigráfico hasta el presente es incierto. Sin embargo, Murphy (1956) encontró a Phylloceras onoense asociado con Gabbieceras wintunium Anderson, a la que asignó al Aptiano (fide Popenoe et. al. 1964, p. 1508).

Los ejemplares de $P$. rioi (= P. velleidae) ilustrados provienen de la misma localidad y unidad estratigráfica que las faunas descritas por Alencáster (1956) Reyeros (1963), Buitrón (1970) por loque se consideran sean del Aptiano.

\section{CONCLUSIONES}

Después del análisis detenido y sin perder de vista los resultados de las comparaciones efectuadas, tanto en los ejemplares disponibles como de las opiniones de los autores oportunamente citados, se concluye que:

1) La autora considera al igual que Wiedmann (1963, p. 213) que las pequeñas diferencias morfológicas no permiten separar $P$. rioi, $P$. onoense y $P$ velladae en especies diferentes.

2) En virtud de la similitud morfológica entre $P$. rioi y $P$. onoense y que ambas especies tienen la misma distribución estratigráfica, se determina que ambas especies son conspecíficas y así $P$. rioi (Nyst y Galeotti), tiene prioridad sobre $H$. onoense (Stanton), por lo que se debe considerar como sinónimo primario de esta última especie. 
3) y para finalizar, habiendo hecho la comparación morfológica se llega que rioi y onoense son sinónimos de velledae, y que hay una pequeña discrepancia, en cuanto a la distribución estratigráfica de los ejemplares tipos de cada una de las especies. Esta diferencia muy probablemente se trate únicamente de problemas de nomenclatura estratigráfica ya que algunas veces los límites Aptiano-Albiano es problemática y por olra parte la posición cronoestratigráfica del Vraconiano es incierta.

Pero el análisis morfológico detallado, realizado por la presente autora, revela que sin duda se trata de un caso de sinonimia. Sin embargo tambien dehe considerarse que la especie en cuestión, tenga un alcance estratigráfico del Aptiano-Albiano hasta el Cenomaniano, Collignon (1949, p. 44) hace notar que $P$. velledae es conocida de todos esos pisos.

\section{BIBLIOGRAFIA CITADA}

AGUILERA, J. G.

1906. Excursión de Tehuacán a Zapotitlán y San Juan Raya México. $\mathrm{X}^{\circ}$ Congr. Geol. Intern. Libreto-guia 7: 1-27; 1 mapa, 1 tabla.

—, ORDOÑEZ, E. y BUELNA, R. J. 1896. Bosquejo Geológico de México. Inst. Geol. México Bol. 4-6: 83-93.

ALENCASTER, G.

1956. Pelecípodos y Gasterópodos del Cretácico Inferior de la Región de San Juan Raya-Zapotitlán, Estado de Puebla. Univ. Nal. Autón, México Inst. Geol. Paleont. México, 2: 1-47, lám. 1-7.

ANDERSON, F. M.

1938. Lower Cretaceous Deposits in California and Oregon. Geol. Soc. America Spec. Pap. 16: 1-329, p. 1-84.

ARKELL, W. J.; KUMMEL, B., y WRIGHT, C. W. 1957. Mesozoic Ammonoidea. in Treatise on Invertebrate Paleontology, Part L, Mollusca. Geol. Soc. America \& Univ. Kansas Press: L1-490, 558 figs.

BOSE, E.

1923. Algunas faunas Cretácicas de Zacatecas, Durango y Guerrero. Inst. Geol. México Bol. 4 1-219 lám. 1-19.

\section{BUITRON, B.}

1970. Equinoides del Cretácico inferior de la Región de San Juan Raya-Zapotitlán, Estado de Puebla. Univ. Nal. Autón. México, Inst. Geol. Paleont. Méxic. 30: 1-45, láms. $1-9$,

BURCKHARDT, C.

1930. Etude Sinthétique sur le Mesozoique Mexicain. Soc. Paleont. Suisse, Mem. 49-50: 1-280; text-figs. 1-32, 11 tablas.

CALDERON, G. A.

1956. Bosquejo Geológico de la región de San Juan Raya. XX Congr. Geol. Intern. Libreto-Guía, Excursión A,11: 9-27.

COLLIGNON, M.

1948. Faune Néocomienne des Couches a Criocéras de
Belohasifaka (Cercle de Sitampiky) (Madagascar). Geol. Serv. Mines Madagascar, Ann. 15: 55-83 1, lám. 8.13, text. figs. $1-8$.

1949. Recherches sur les Faunes Albiennes de Madagascar. 1..- D'Albien D'Ambarimaninga. Ann. Geol. Serv. Mines, Madagascar, 15, :5-119, lám. 1-20, text figs. 1-29.

COQUAND, $\mathrm{H}$. 1869. Monographie du genre Ostrea. Terrain Crétacé :1215, láms. 1-75.

COTTEAU, G. H.

1890. Note sur quelques Echinides du Terrain Crétacé $d u$ Mexique Bull. Soc. Géol. France. Ser. 3, 8 :292-299, lám. 1-2

DESOR, E. 1855-1858. Synopsis des échinides fossiles Paris, :1-490, láms. 1-44.

GABB, W. M. 1864. Description of the Cretaceous Fossils. Geol. Surv. California, Paleont, 1 :57-217, lám. 9-32.-

HAAS, OTTO 1942. The Vernay Collection of Cretaceous (Albian) Ammonites from Angola. American Mus. Nat. Hist., Bull. 81 :1-294, text-fig. 1-34, láms. 1-48, 3 tablas.

HANNA, G. D. Y HERTLEIN, L. G. 1943. Characteristic Fossils of California. State California, Div. Mines, Bull. 118 :165-182, text-fig. 60-67.

MALDONADO-KOERDELL, M. 1953. Los equinoides regulares del Mesozoico de México An. Esc. Nac. Cienc. Biol. 7, 1-4- :15-44, lám. 1-2.

MATSUMOTO, TATSURO 1959. Upper Cretaceous ammonites of California. Kyushu Univ., Mem. Fac. Sci., ser. (D), spec. 1 (2) :1-172; láms. 1-41.

MICHELIN, H. 1834. Magazine de zoologie, lám. 35 (teste d'Orbigny). MULLERRIED, F. K. G. 1933. Estudios Paleontológicos y Estratigráficos en la Región de Tehuacán Puebla, México (Segunda Parte). Inst. Biól. Univ. Nal. México, An. 4, 2:79-83, fig. 7-14.

1934. Estudios Paleontológicos y Estratigráficos de Tehuacán Puebla, México (Conclusión). Inst. Biól. Univ. Nal. México, An. 5, 1 :75-80; fig. 34-39.

MURPHY, M. A. 1956. Lower Cretaceous Stratigraphic Units of Northern California. American Assoc. Petrol. Geol., Bull. 40 :20982119, figs. 1-6.

NYST, H. Y GALEOTTI, H. 1940-41. Description des Mollusques et Rayonnés Fossiles. Tehuacán au Mexique. Royal Acad. Bruxelles, Bull. 7: 212-221, láms. 1-2.

ORBIGNY, A. d' 1840-41, Description des Mollusques et Rayonnés Fossiles, Terrains Cretacés (Cephalopodes) Paris. Paleont. Univers. Francaise 1 :1-662; láms. 1-148.

1850. Prodrome de Paleontologie Stratigraphique Universelle. Paris. 2 :1-427.

PACKARD, E. L. 1960. Hypotypes of Phylloceras onoense Stanton, Jour. Paleont. 34 :421.428, text-fig. 1-2, láms. 55--517.

PICTET, F. J. y RENEVIER, E. 1858. Description des fossiles del Terrain Aptien de la 
Parte du Rhone et des Voirons de Ste-Croix. Paleont. Suisse ;1-183, lám. 1-23.

POPENOE, W., IMLAY, R. W., y MURPHY, M. A.

1960. Correlation at the Cretaceous Formations of the Pacific Coast (United States and Northwestern Mexico).

Geol. Soc. America. Bull. 71 :1491-1540, 1 tabla.

REYEROS, M.

1963. Corales del Cretácico Inferior de San Juan Raya, Estado de Puebla. Univ. Nal. Autón. México., Inst. Geol. Paleont. Mexic. 17 :1-21, lám. 1-5.

SMITH, P. J.

1898. The Development of Lyloceras and Phyiloceras. California Acad. Sci., Proc. Ser. (3 1, 4 :129-150, láms. 16-20.
SPATH, L. F. 1927. Revision of the Jurassic cephalopod Fauna of Kacch (Cutch). Geol. Surv. Mem. Paleont. Indica, n. ser. (9), 2 :1-71, pl. 1-7.

STANTON, T. W.

1955. Contributions to the Cretaceous paleontology of the Pacific Coast. The fauna of the Knoxville beds. U. S. Geol. Surv., Bull. 133 :1-32, pl. 1-7.

WIEDMANN,J.

1963. Unterkreide-Ammoniten von Mallorca, 2. Lieferung: Phylloceratina. Ak. Wiss. Lit., Abh. Math. Nat. Kl. 4:151-256, fig. text 1-64, lám. 11-21. 


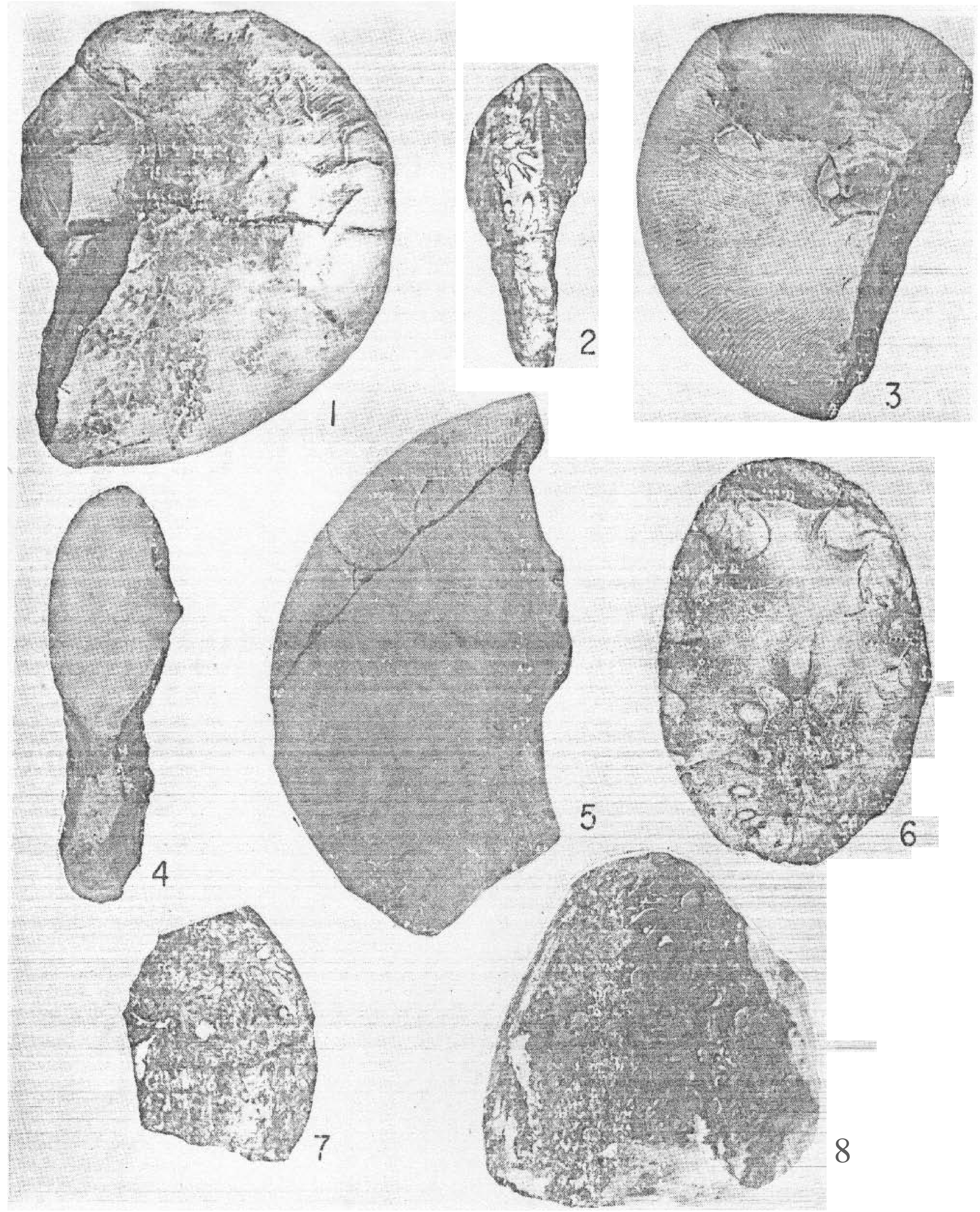

Figuras 1-8 Phylloceras (Hypophilloceras) velledae (Michelin 1834).

Figs. 4 y 6 Sección de vuelta de los fragmentos IGM 2534 y IGM 2533 respectivamente

Fig. 5.- Hipotipo IGM 2529.

Figs. 1 y 3.-Hipoticos IGM 2530 (X0.75) IGM respectivamente.

Fig. 8.-Fragmento con sutura IGM 2532. 